\title{
Non-invasive Estimation of Pressure Changes using 2-D Vector Velocity Ultrasound: An Experimental Study with In-Vivo Examples
}

Olesen, Jacob Bjerring; Villagómez Hoyos, Carlos Armando; Møller, Niclas Dechau; Ewertsen, Caroline; Hansen, Kristoffer Lindskov; Nielsen, Michael Bachmann; Bech, Bo; Lönn, Lars; Traberg, Marie Sand; Jensen, Jørgen Arendt

\section{Published in:}

IEEE Transactions on Ultrasonics, Ferroelectrics and Frequency Control

Link to article, DOI:

10.1109/TUFFC.2018.2808328

Publication date:

2018

Document Version

Peer reviewed version

Link back to DTU Orbit

Citation $(A P A)$ :

Olesen, J. B., Villagómez Hoyos, C. A., Møller, N. D., Ewertsen, C., Hansen, K. L., Nielsen, M. B., Bech, B.

Lönn, L., Traberg, M. S., \& Jensen, J. A. (2018). Non-invasive Estimation of Pressure Changes using 2-D Vector Velocity Ultrasound: An Experimental Study with In-Vivo Examples. IEEE Transactions on Ultrasonics,

Ferroelectrics and Frequency Control, 65(5), 709-719. https://doi.org/10.1109/TUFFC.2018.2808328

\section{General rights}

Copyright and moral rights for the publications made accessible in the public portal are retained by the authors and/or other copyright owners and it is a condition of accessing publications that users recognise and abide by the legal requirements associated with these rights.

- Users may download and print one copy of any publication from the public portal for the purpose of private study or research.

- You may not further distribute the material or use it for any profit-making activity or commercial gain

- You may freely distribute the URL identifying the publication in the public portal 


\title{
Non-invasive Estimation of Pressure Changes using 2-D Vector Velocity Ultrasound: An Experimental Study with In-Vivo Examples
}

\author{
Jacob Bjerring Olesen*, Carlos Armando Villagómez-Hoyos*, Niclas Dechau Møller*, Caroline Ewertsen ${ }^{\dagger}$, \\ Kristoffer Lindskov Hansen ${ }^{\dagger}$, Michael Bachmann Nielsen ${ }^{\dagger}$, Bo Bech ${ }^{\dagger}$, Lars Lönn ${ }^{\dagger}$, \\ Marie Sand Traberg*, and Jørgen Arendt Jensen* \\ ${ }^{*}$ Center for Fast Ultrasound Imaging, Technical University of Denmark, DK-2800 Kgs. Lyngby, Denmark. \\ ${ }^{\dagger}$ Department of Diagnostic Radiology, Rigshospitalet, DK-2100 Copenhagen, Denmark.
}

\begin{abstract}
A non-invasive method for estimating intravascular pressure changes using 2 -D vector velocity is presented. The method was first validated on computational fluid dynamics (CFD) data, and with catheter measurements on phantoms. Hereafter, the method was tested in-vivo at the carotid bifurcation and at the aortic valve of two healthy volunteers. Ultrasound measurements were performed using the experimental scanner SARUS, in combination with an $8 \mathrm{MHz}$ linear array transducer for experimental scans and a carotid scan, whereas a $3.5 \mathrm{MHz}$ phased array probe was employed for a scan of an aortic valve. Measured 2-D fields of angle-independent vector velocities were obtained using synthetic aperture imaging. Pressure drops from simulated steady flow through six vessel geometries spanning different degrees of diameter narrowing, running from $20 \%-70 \%$, showed relative biases from $0.35 \%$ to $12.06 \%$, depending on the degree of constriction. Phantom measurements were performed on a vessel with the same geometry as the $70 \%$ constricted CFD model. The derived pressure drops were compared to pressure drops measured by a clinically used $4 \mathrm{~F}$ catheter and to a finite element model. The proposed method showed peak systolic pressure drops of $-3.0 \mathrm{kPa} \pm 57 \mathrm{~Pa}$, while the catheter and the simulation model showed $-5.4 \mathrm{kPa} \pm 52 \mathrm{~Pa}$ and $-2.9 \mathrm{kPa}$, respectively. An in-vivo acquisition of $10 \mathrm{~s}$ was made at the carotid bifurcation. This produced eight cardiac cycles from where pressure gradients of $-227 \mathrm{~Pa} \pm 15 \mathrm{~Pa}$ were found. Lastly, the aortic valve measurement showed a peak pressure drop of $-2.1 \mathrm{kPa}$ over one cardiac cycle. In conclusion, pressure gradients from convective flow changes are detectable using 2-D vector velocity ultrasound.
\end{abstract}

\section{INTRODUCTION}

Abnormal changes in intravascular blood pressure are an indication of vascular disease. Measuring pressure variations is therefore used clinically as a diagnostic marker, when assessing the pathological state of a cardiovascular region [1]. Intravascular pressure is currently assessed by inserting pressure sensing wires or catheters into the brachial or femoral artery, and threading them to the region of interest. After reaching the desired region, the lesional pressure drop $\Delta P$, is registered by measuring proximally and distally to the lesion. The difference in pressure between the two locations gives the relative change in pressure. Despite the advantage offered by a pressure catheter in terms of low inter-operator dependency, the procedure suffers some severe limitations as it is highly invasive and requires the use of ionizing radiation for guidance in an angio suite. Furthermore, a recent report from De Vecchi et al. [2] demonstrated that the accuracy of catheters depends on the size and shape of the catheter. A $24 \%$ overestimation of the peak systolic pressure was found, when using a routinely employed catheter compared to a pressure sensing wire, which is considered the gold standard [2], [3].

The motivation to assess intravascular pressure variations using less-invasive approaches appeared in the 1970s. In 1977, Fairbank and Scully [4] proposed a method for estimating local pressure changes using microbubbles. The suggested method relied on injecting gas-filled bubbles into the vascular system and measuring the frequency shift that occurred in the scattered spectrum as ultrasonic waves were applied. The idea of using microbubbles for obtaining estimates of pressure led the way for a range of methods devised from this technique [5]-[8]. Despite the less invasiveness of these procedures, they still required the injection of microbubbles.

In 1976, Holen et al. [9] introduced the first fully noninvasive alternative for estimating intravascular pressure based on Doppler ultrasound. Analysing audio signals of the frequency shifts received from the mitral jet, revealed the peak systolic velocity. From this, the local pressure gradients were calculated using an orifice equation. The usefulness of the method was demonstrated on 25 patients with mitral stenosis and 10 without. The method was attractive due to the avoidance of catheterization, but was faced with difficulties associated with ultrasound scanner technology of that time, e.g. poor signal-to-noise ratios, and inferior temporal and spatial resolutions. However, the dominant drawback of the method was its reliance on a single velocity estimate, which made the method highly sensitive to hemodynamic factors unrelated to the constricted vessel's effect on the peak velocity, e.g. abnormal cardiac output [10]. The method presented by Holen et al. was later tested against clinical pressure catheters by Strauss- and Baumgartner et al. [11]-[13]. The latter studies agreed that non-invasive pressure estimation through a simplified version of the Bernoulli equation was achievable. However, the obtained pressure estimates were greatly dependent on the size of the examined vessel, and the examiners ability to correct for the Doppler angle. Further advancement in non-invasive techniques for improving pressure estimates have been proposed over the past decades [14]-[25]. Though, many of which remain limited by the crude 1-D 
approximation made for most flow estimators that are available in the clinic.

In this paper, a method for estimating pressure changes from 2-D vector velocity ultrasound data using the nonsteady Bernoulli equation is proposed. Firstly, the method is tested on finite element flow data, and the results are compared with a priori known pressure fields. Next, experimental scans are performed on a flow phantom with a concentric diameter constriction of $70 \%$ according to NASCET 1991 [26]. Here, the derived changes in pressure are compared with results from a routinely employed pressure catheter as well as from simulation models. The study concludes by testing the method in-vivo at the carotid bifurcation and at the aortic valve in two healthy volunteers.

\section{BACKGROUND}

Blood moving through an arterial stenosis experiences a drop in pressure. This drop is a consequence of primarily three factors: fluid viscosity, turbulent flow features, and forced convection from luminal narrowing. Viscous energy loss leads to a pressure drop as mechanical energy is converted into heat. This is seen both in the fluid-wall interaction, but also between adjacent layers of fluid moving at different velocities. The dissipation of energy into heat is also the reason, why pressure drops are seen in turbulent flow environments. Here, small eddies form as the flow passes an obstacle, and that dissipates energy. Lastly, changes in the pressure will rise from spatial changes in flow velocities as the blood moves through the diseased area. Whether the pressure drop is dominated by viscous, or turbulent energy losses, or by forced convection is entirely dependent on the volume flow, and on the geometrical shape of the vessel. For instance, in small vessels such as capillaries, the flow is slow, which creates an environment where pressure losses primarily are governed by frictional forces. Moving into larger vessels with increasing flow velocities, such as the carotid arteries or the aorta, the effect of viscosity diminishes, and the drop in pressure is dictated by convective and turbulent energy losses. The split between convective and turbulent energy losses is greatly dependent on the geometry of the vessels lumen. This study concerns a model that relies on vector velocity data to determine pressure changes caused by convective flow effects, thus, the viscous and turbulent terms are neglected. The consequence of this is discussed in not all factors are taken into account. Whether this is reasonable is discussed in Section VIII.

\section{Pressure Changes from Vector Velocity Flow}

The Navier-Stokes equations presuming the conservation of mass and linear momentum is:

$$
\rho\left[\frac{\partial \vec{v}}{\partial t}+\vec{v} \cdot \nabla \vec{v}\right]=-\nabla p+\rho \vec{g}+\mu \nabla^{2} \vec{v} .
$$

It describes the development of a fluid's velocity field $\vec{v}(\vec{r}, t)=\left(v_{x}(t), v_{y}(t), v_{z}(t)\right)$ by relating the forces acting on an incompressible volume to its acceleration and density throughout time, $t$, and space, $\vec{r}$. The left-hand side sums the local $\frac{\partial \vec{v}}{\partial t}$ and convective fluid acceleration $\vec{v} \cdot \nabla \vec{v}$, where $\rho$ is the density of the fluid. The right-hand side sums the surface and volume forces acting on the fluid. The forces constitute a pressure drop $-\nabla p$, a gravitational force $\vec{g}$, and a viscous drag caused by the viscosity of the fluid $\mu \nabla^{2} \vec{v}$. The gravitational term is usually neglected, as a patient undergoing an ultrasound scan is placed in a supine position. Further, Prantdl and Wood [27], [28] showed that the effect of the viscous term is neglectable, when studying blood flow in larger arteries, as it only has a small influence on the overall movement of flow. This is especially true in the aortic valve or across severe constrictions, where significant flow changes arise from drastic alterations in the cross-sectional area [29]-[31].

A full view of the relative intravascular pressure distribution can be mapped through solving a least-square approximation of the derived pressure gradient field, $-\nabla p$, using the PressurePoisson equation [32], [33]. However, this has been shown to be greatly dependent on an accurate segmentation of the vessel and its boundaries to set the necessary boundary conditions needed for solving the pressure equation [34], [35]. Instead, a different approach of deriving pressure is considered. Vector velocity imaging allows tracking of blood scatterers along tortuous streamlines from where pressure changes can be derived. Rewriting (1) into a scalar equation following a streamline, and omitting the influences of gravity and viscosity, yields the following;

$$
\mathrm{d} p_{s}=-\rho\left[\frac{\partial v_{s}}{\partial t} \mathrm{ds}+v_{s} \frac{\partial v_{s}}{\partial s} \mathrm{ds}\right] .
$$

Here, $v_{S}$ is the scalar product of $\vec{v}(\vec{r}, t)$ and the unit vector that lies tangential to the streamline $d \vec{s}=\left(\hat{s}_{x}, \hat{s}_{y}, \hat{s}_{z}\right)$, where $d \vec{s}$ is an element of distance along the streamline that runs in the direction $s$. In an isolated frame of vector velocities, $v_{s}$ is assumed to vary only as a function of $s$, thus, $d s \approx \partial s$. Then, integrating the individual pressure gradients $\mathrm{d} p_{s}$ along the flow direction of the streamline from point $l_{1}$ to $l_{2}$ gives the summed pressure drop $\Delta p$, between these points of the streamline,

$$
\Delta P(t)=\int_{l_{1}}^{l_{2}} \mathrm{~d} p_{s}(t)=p(t)_{s, l_{2}}-p(t)_{s, l_{1}},
$$

or

$$
\Delta P(t)=-\rho\left[\frac{v_{s, l_{2}}^{2}-v_{s, l_{1}}^{2}}{2}+\int_{l_{1}}^{l_{2}} \frac{\partial v_{s}}{\partial t} \mathrm{ds}\right] .
$$

Eq. (4) is also referred to as the nonsteady Bernoulli equation [15]. Further, (2)-(4) state that the three spatial vector components of $\vec{v}$ must be known to estimate the streamline pressure gradient $\mathrm{d} p_{s}$, as the flow moves in a 3-D volume. This study employs a velocity estimator, which yields the twodimensional (2-D) in-plane vector velocity field $\vec{v}=\left(v_{x}, v_{z}\right)$. The proposed method is, thus, investigated assuming that the out-of-plane velocity $v_{y}$ is zero.

\section{EXPERIMENTAL SET-UP AND DATA ACQUISITION}

The performance of the proposed method was investigated on simulated flow data with a priori knowledge of the pressure 
field, followed by flow phantom experiments. The precision of the method was tested on in-vivo data acquired by a radiologist for two healthy volunteers.

Finite element (FE) simulations of flow through six flow geometries each having a different degree of constriction were designed. The geometry for each vessel was drafted in SolidWorks (Education edition, Dassault Systèmes SolidWorks Corp., Vélizy, France), and constrictions varied from $20 \%$ to $70 \%$. The drafted models were exported in a STEP format to Comsol (Comsol Multiphysics v5.2a, Comsol AB, Stockholm, Sweden). Here, flow was simulated using an in-built PARDISO solver with an outlet pressure of $0 \mathrm{~Pa}$ and a no-slip condition at the wall. The simulations were made in laminar flow regimes. Further, the properties of the emulated fluid were set to match those of the experimental used blood-mimicking fluid, e.g. a viscosity of $4.1 \times 10^{-3} \mathrm{Pas}$ and a density of $1030 \mathrm{~kg} / \mathrm{m}^{3}$.

A flow phantom was designed and fabricated, such that it had the same fluid domain as the FE model with the $70 \%$ constriction equivalent to an area reduction of $90 \%$. Similar flow domains were achieved by exporting the original drafted model to stereolithography on an Ultimaker $2+$ printer (Ultimaker, Geldermalsen, Netherlands), with a depth-width resolution of $12.5 \mu \mathrm{m}$ and a resolution in height of $5 \mu \mathrm{m}$. The $3-D$ printed geometry then constituted the core in a wallless flow phantom using the fabrication process developed by Lai et al. [36]. The core was fixated in a container before casting it in polyvinyl alcohol (PVA) cryogel to make the surrounding medium mimic the properties of human tissue. The PVA cryogel contains 15\% PVA, 3\% graphite, $0.3 \%$ potassium sorbate, and $81.7 \%$ distilled water. The nature of cryogel allows for controlling its elastic properties by varying the number of freeze-thaw cycles it undergoes. Three freezethaw cycles were used, each half-cycle of 24-hours duration, with freeze settings of $-20{ }^{\circ} \mathrm{C} \pm 0.5^{\circ} \mathrm{C}$ and thaw of $4{ }^{\circ} \mathrm{C} \pm$ $0.5^{\circ} \mathrm{C}$. After completing three cycles, given a total duration of $144 \mathrm{~h}$, the core was removed manually, leaving a core-less phantom with a fluid domain identical to the $70 \%$ constricted FE model.

The fabricated phantom was connected to a flow system (CompuFlow 1000, Shelley Medical Imaging Technologies, Toronto, Canada) that circulated a blood-mimicking fluid (BMFUS, Shelley Medical Imaging Technologies, Toronto, Canada) with a viscosity of $4.1 \times 10^{-3} \mathrm{Pas}$ and a density of $1030 \mathrm{~kg} / \mathrm{m}^{3}$. Initially, a constant volume flow of $15 \mathrm{~mL} / \mathrm{s}$ was applied to the phantom. Secondly, a time-varying waveform was imposed having a peak flow rate of $15 \mathrm{~mL} / \mathrm{s}$, and a cycle period of $0.84 \mathrm{~s}$. This gave a cycle flow of $3.85 \mathrm{~mL}$, and an average flow rate of $275 \mathrm{~mL} / \mathrm{min}$, which is within the normal physiological range of the carotid artery [37].

\section{Direct pressure estimation using a catheter}

The reference pressure for validating the experimentally derived pressure estimates was obtained using a clinically employed 4F Berenstein catheter (Soft-Vu, Angiodynamics, NY, USA) connected to a standard pressure transducer (TruWave, Edward Lifesciences, CA, USA). The catheter was introduced to the circulating flow system through an $11 \mathrm{~F}$ arterial sheath
(Cordis Corporation, FL, USA), which was mounted onto a three-way junction placed before the flow phantom. The tip of the catheter was guided by ultrasound using a BK Ultrasound 5000 scanner (BK Ultrasound, Herlev, Denmark). Pressure data were sampled using a DAQ card (PCIe 6251, National Instruments, TX USA) at a frequency of $400 \mathrm{~Hz}$. A bioamplifier (ETH-256, CB Sciences, NH USA) provided the input excitation voltage for the transducer (10 V DC) as well as amplification of the output signal $(G=1000)$. Recordings were performed in LabView (National Instruments, TX, USA).

\section{Vector velocity estimation}

Fields of vector velocities were estimated using directional synthetic aperture flow imaging, an ultrasound approach developed by Villagómez-Hoyos et al. [38], [39]. Here, a low-resolution image is created for each emission, and multidirectional lines are beamformed for every position within the insonified area. The flow angle and its velocity are found by cross-correlating beamformed lines to lines in successive low resolution images, allowing a frame-rate close to the pulse repetition frequency.

Both phantom and the in-vivo scan of the carotid artery were made using a 128-element linear array probe connected to the experimental research scanner SARUS [40]. A threecycle pulse with a center frequency of $8 \mathrm{MHz}$ was emitted at an acquisition frequency of $12.5 \mathrm{kHz}$ to a depth of $4.5 \mathrm{~cm}$. Five low-resolution images were summed for each high resolution image, which potentially could provide a frame-rate of $2.5 \mathrm{kHz}$. An acquisition frequency in kilohertz is required for estimating flow velocities that are in several meters per second, otherwise the signal between successive emissions gets too decorrelated. The frame-rate on the other hand is not required to be in kilohertz as long as all temporal flow changes are captured throughout the cardiac cycles. Thus, to minimize the computational load, flow images corresponding to a frame-rate of $350 \mathrm{~Hz}$ were processed by skipping frames. For the cardiac scan, a 128-element phased array probe was used with a center frequency of $3.5 \mathrm{MHz}$. Here, a scan-depth of $11 \mathrm{~cm}$ was used, and the acquisition frequency was lowered to $8.5 \mathrm{kHz}$. The number of summed low-resolution images was reduced to three to cope with the high velocities found across the heart valves. In this case, all of the acquired data were beamformed producing a frame-rate of roughly $2830 \mathrm{~Hz}$. The collected ultrasound data were processed off-line using Matlab (The MathWorks Inc., Natick, MA, USA).

The in-vivo acquisitions were carried out after approval by The Danish National Committee on Biomedical Research Ethics (approval number H-1-2014-FSP-072). The carotid bifurcation of a healthy male (age 27) and the aortic valve of another healthy male (age 30) were scanned by an experienced radiologist $(\mathrm{KLH})$. The volunteers were placed in a supine position for five minutes to obtain a steady heart rate. The carotid scan was acquired in a long axis view over the bifurcation, while the aortic valve was scanned in long axis view parasternally. Intensity measurements were made prior to the examinations, showing mechanical indexes of 0.91 and 0.62 , and $I_{\text {spta }} \mathrm{s}$ of $144.3 \mathrm{~mW} / \mathrm{cm}^{2}$ and $106.8 \mathrm{~mW} / \mathrm{cm}^{2}$ for the carotid 


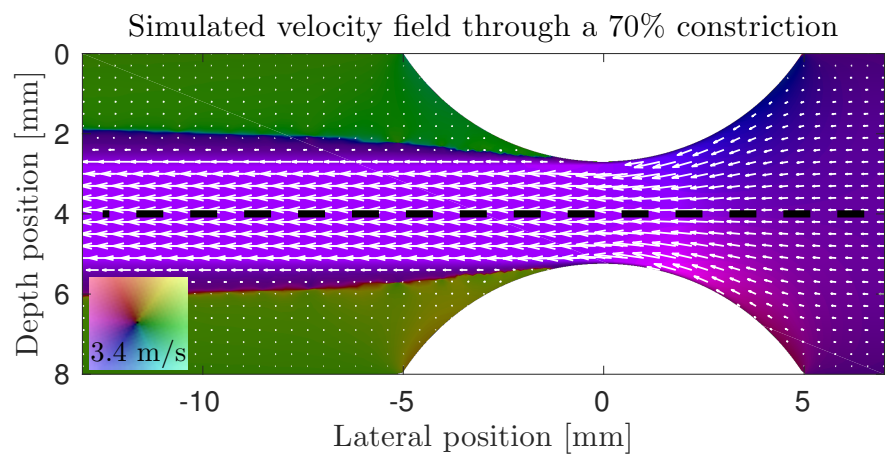

Fig. 1. Simulated vector velocity map of constant flow through the $70 \%$ constricted phantom. The dotted line indicates a streamline that has its starting point right of the constriction.

and the cardiac scan, respectively. They were, thus, within the limits set by the U.S. Food and Drug Administration [38], [41].

\section{Simulation Results}

Flow simulations through the six geometries were made, and the velocity/pressure data were extracted for testing the performance of the proposed method in a controlled environment. Initially, constant flow was studied with a volume rate of $15 \mathrm{~mL} / \mathrm{s}$, before the method was tested in a time-varying flow environment.

\section{Simulated constant flow}

The result of simulating vector flow over the $70 \%$ constricted model is shown in Fig. 1. The image shows flow that accelerates toward the center of the constriction, producing a jet that slowly decelerates as the lumen expands again. Superimposed onto the image is a dashed line representing a streamline set to start at the lateral position of $7 \mathrm{~mm}$. The position of the streamline was selected, so that it originates from the center of the vessel and stops as it crossed the boundaries of the color box. Velocities along the streamline were collected and inserted into (4) for deriving the trans-stenotic pressure drop. The result is shown in Fig. 2 as the orange line along with the simulated pressure drop from the FE model (dotted line). Studying the pressure drop across the $70 \%$ constriction reveals a peak pressure drop of $-5.4 \mathrm{kPa}(-40.5 \mathrm{mmHg})$ and a discrepancy of $0.7 \%$ between the FE model and the results of applying (4) onto the simulated flow data.

The result of repeating this comparison for all of the designed geometries are shown in Fig. 3. Here, the flow rate is kept fixed at $15 \mathrm{~mL} / \mathrm{s}$, while the degree of the constriction changes. The figure shows a decrease in error as the constriction grows from $20 \%$ to $70 \%$. The reason for this is found in the balance between pressure changes due to frictional forces and pressure changes due to forced convection of the flow. As the constriction grows, the flow velocity increases, thus, making convective forces significantly more prominent than the forces arising from viscosity. This is also confirmed by examining the Reynolds number for these six cases, which present values ranging from 800 to 2,000 suggesting that the majority of flow
Simulated data: Pressure estimator vs. finite element

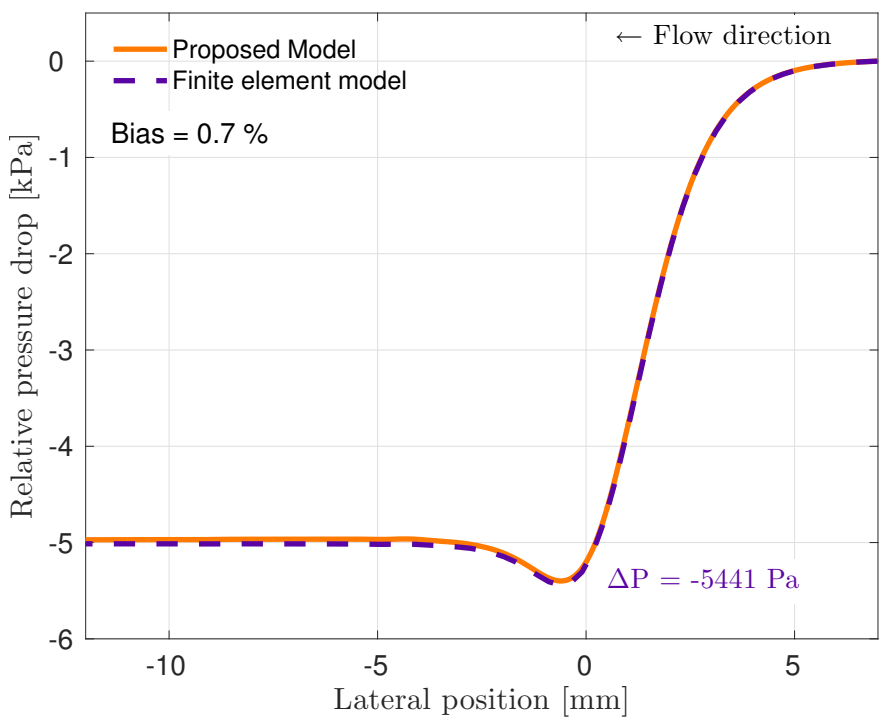

Fig. 2. Pressure drop along the streamline from Fig. 1. The estimated drop is shown together with the result from the FE model.

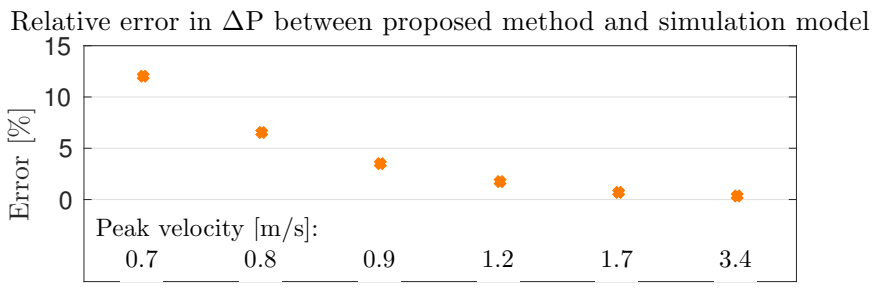

Vessels with varying degree of constrictions

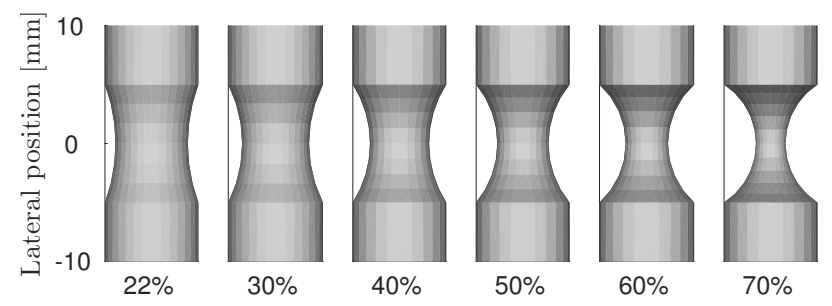

Fig. 3. Discrepancies between the proposed model and the FE simulations in varying flow geometries, when comparing the peak pressure drop across the constrictions. The top graph shows the error and the bottom figure illustrates the associated geometry.

is governed by inertial forces. However, increased velocities may raise a new concern, as flow at higher velocities tends to become more prone to turbulent flow, which is not included in the estimator.

\section{Simulated pulsating flow}

Time-varying flow through the $70 \%$ constricted geometry was simulated and used for testing the performance of the estimator. A waveform having a stroke volume flow of $3.85 \mathrm{~mL} / \mathrm{s}$ was applied to the inlet of the FE simulation. Then, using the proposed estimator on the simulated velocities yields the results in Fig. 4. Here, the surface plot presents pressure changes over both time and lateral streamline position. The bottom plane reveals the bias between estimated pressure changes and the reference model, which for the concerned 


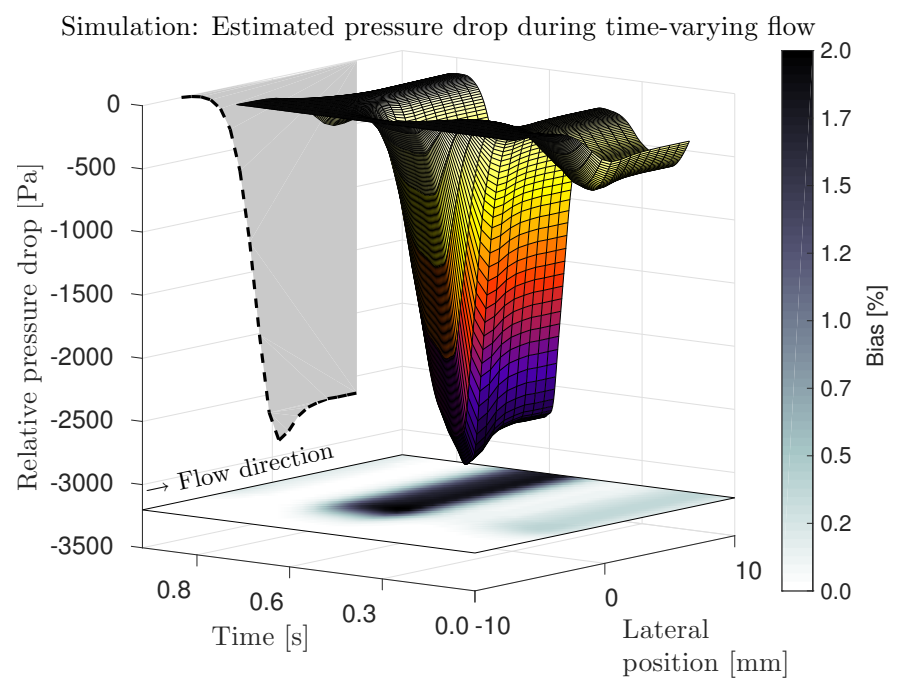

Fig. 4. Pressure drop estimated from the simulated velocities during time-varying flow. The surface plot shows pressure changes as a function of time and lateral position. Bottom plane reveals the bias between estimator and reference model.

geometry lies below $2 \%$ of the peak pressure drop. This indicates that the proposed estimator is capable of deriving pressure changes in both stationary and time-varying flow that are comparable to the output of the FE model.

\section{Flow Phantom Results}

Next, the performance of the method was investigated experimentally on a flow phantom with similar geometry as the $70 \%$ constricted FE model. Measurements were made for steady and pulsating flow at the site of the constriction over acquisition times of $0.23 \mathrm{~s}$, and $10 \mathrm{~s}$, respectively. An example of a single vector velocity frame acquired over the fabricated phantom is displayed in Fig. 5. The estimated peak velocity is slightly underestimated compared to the FE model, $3.0 \mathrm{~m} / \mathrm{s}$ in reference to $3.4 \mathrm{~m} / \mathrm{s}$. This underestimation is likely a result of misalignment of the transducer, so that the actual peak flow is outside of the examined scan plane. This also explains why the flow jet is much more pronounce in case of the CFD model than what is estimated. Misalignment of the transducer to the peak flow, or inherent underestimation of the flow estimator will directly affect the pressure estimates when using (4), as the difference in pressure scales with the magnitude of the squared velocity component.

\section{Constant flow}

The results of estimating pressure change across the constricted phantom during constant flow conditions are displayed in Fig. 6. The graph shows the estimated, simulated, and measured pressure drop that occurs as the fluid moves through the constricted phantom. The shaded zone indicates the region of one standard deviation. The purple diamonds in Fig. 6 are the results from the catheter measurements made prior to the ultrasound acquisition. The standard deviation of the catheters measurements was calculated from an acquisition window of $17 \mathrm{~s}$ sampled at $400 \mathrm{~Hz}$ before, under, and after the constriction.

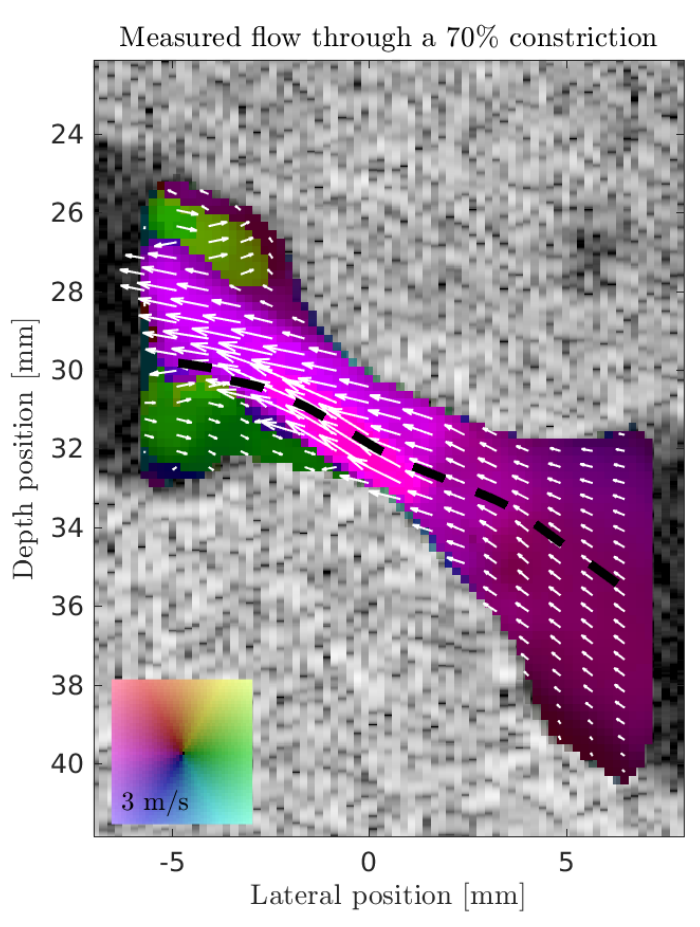

Fig. 5. Vector velocity ultrasound image over the $70 \%$ constriction flow phantoms. The image is captured during steady conditions at a flow rate of $15 \mathrm{~mL} / \mathrm{s}$ with a black-dotted streamline passing through the center of the constrictions.

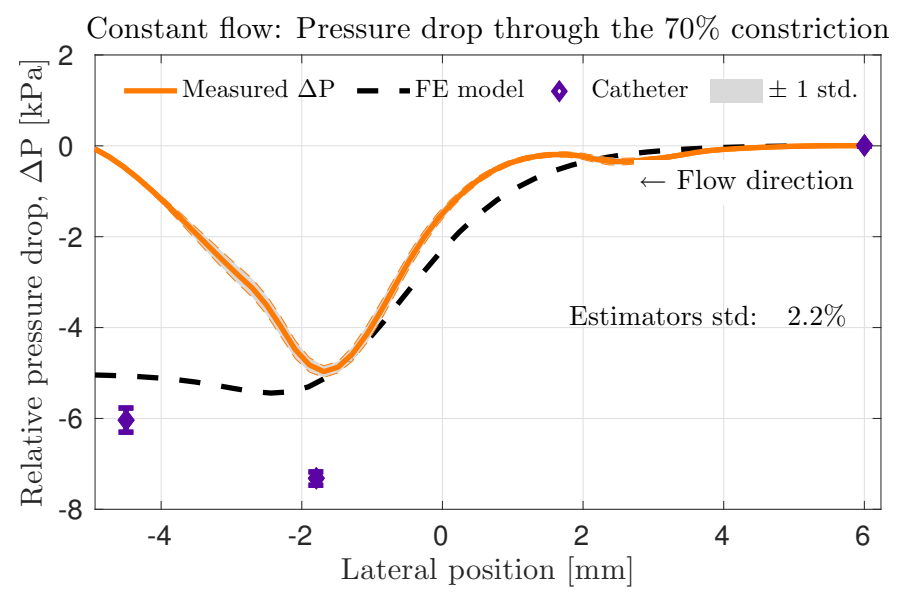

Fig. 6. Constant flow: Estimated, measured and simulated pressure drop through the center of a $70 \%$ constriction. The mean of 70 estimates is plotted together with \pm 1 standard deviation.

The dashed line corresponding to the results from the FE model is also presented in Fig. 2. The relative standard deviations of the proposed model and the catheter measurement, in reference to the peak pressure drop were $2.2 \%$, and $2.0 \%$, respectively. Studying Fig. 6 from right-to-left shows a decent accordance between the estimated and simulated pressure drop upstream to the constriction (lateral positions: $6 \mathrm{~mm}$ to $-2 \mathrm{~mm}$ ), whereas an increasing mismatch arises in the post-stenotic region (lateral positions: $-2 \mathrm{~mm}$ to $-5 \mathrm{~mm}$ ). The increase in pressure seen by the proposed model was similar to what would happen if pressure recovery were present. However, at flow velocities of 


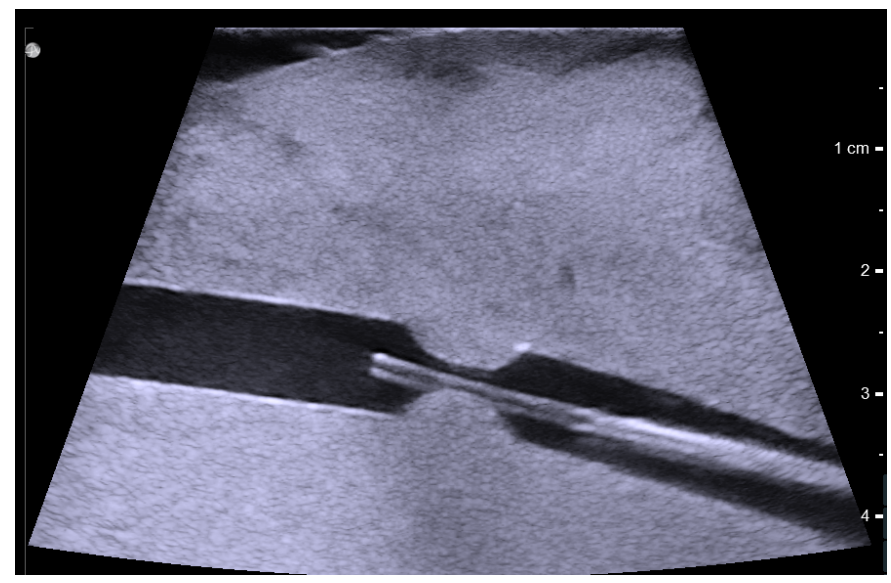

Fig. 7. B-mode image of the fabricated flow phantom having a $4 \mathrm{~F}$ catheters placed after the constriction along the direction of the flow. The image is acquired by the BK5000 scanner, (BK Ultrasound, Herlev, Denmark).

$3.0 \mathrm{~m} / \mathrm{s}$ inside the constriction, it is unrealistic that pressure recovery would already occur immediately after the geometry expands again. Instead, the rise in pressure seen by the proposed method is the result of having angulated the transducer, so that the scan plane missed capturing the jet that forms after the constriction.

Catheter measurements were also acquired during constant flow, and a peak pressure drop of $-7.3 \mathrm{kPa} \pm 147 \mathrm{~Pa}$ was measured $(-54.8 \mathrm{mmHg} \pm 1.1 \mathrm{mmHg})$. In reference to the simulations, the catheter overestimated by $34 \%$. This overestimation is due to the bulk size of the catheters relative to the luminal area, which influences the pressure it intends to measure. An example of the catheter's physical presence inside the constricted phantom is displayed in Fig. 7.

\section{Pulsating flow}

The result of repeating the experiment in a pulsating flow environment is displayed in Fig. 8. The figure shows the estimated, simulated, and measured pressure drop occurring in peak systole, as changes here were the most prominent. The estimated peak pressure drop was calculated from eight full flow cycles. The precision of both the estimator and the catheters was equivalent to that of the steady flow experiment with standard deviations of $1.9 \%$, and $1.0 \%$, respectively. However, for this setup the scan plane was aligned to the jet of the flow producing better accordance between the estimated and simulated pressure drop. The bias of the estimator was found to $7.1 \%$ of the peak simulated pressure drop, when compared to the CFD model. The catheter measured a pressure drop of $-5.4 \mathrm{kPa} \pm 52 \mathrm{~Pa}(-40.5 \mathrm{mmHg} \pm 0.4 \mathrm{mmHg})$ over 70 flow cycles, giving a bias of $85.1 \%$ in comparison to the CFD model.

A summary of the results from the experimental study is listed in Table I. The listed values were found across the length of the constriction.

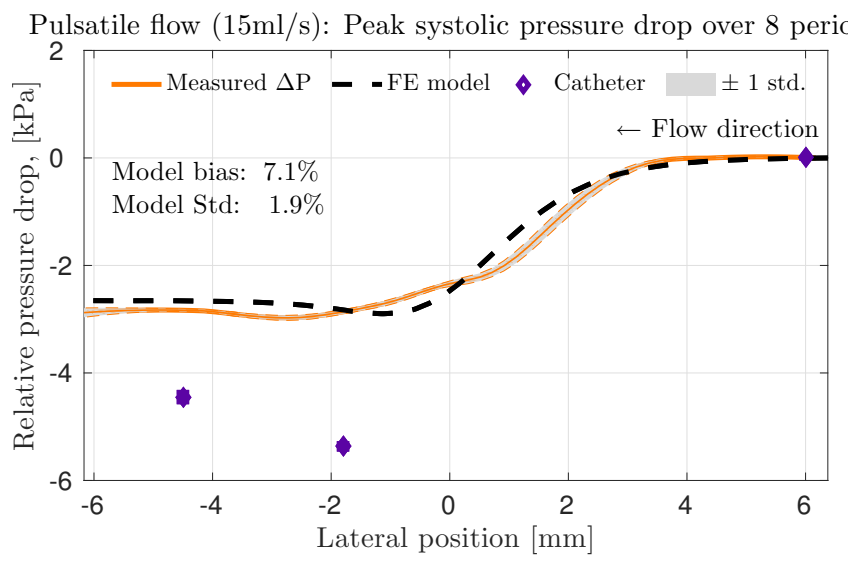

Fig. 8. Pulsatile flow: The average peak pressure drop through the center of a $70 \%$ constriction during a peak flow of $15 \mathrm{~mL} / \mathrm{s}$. The average was found over eight successive cycles, and compared with the pressure drop measured directly by the catheter during the same phase of the flow cycle, and with the finite element model.

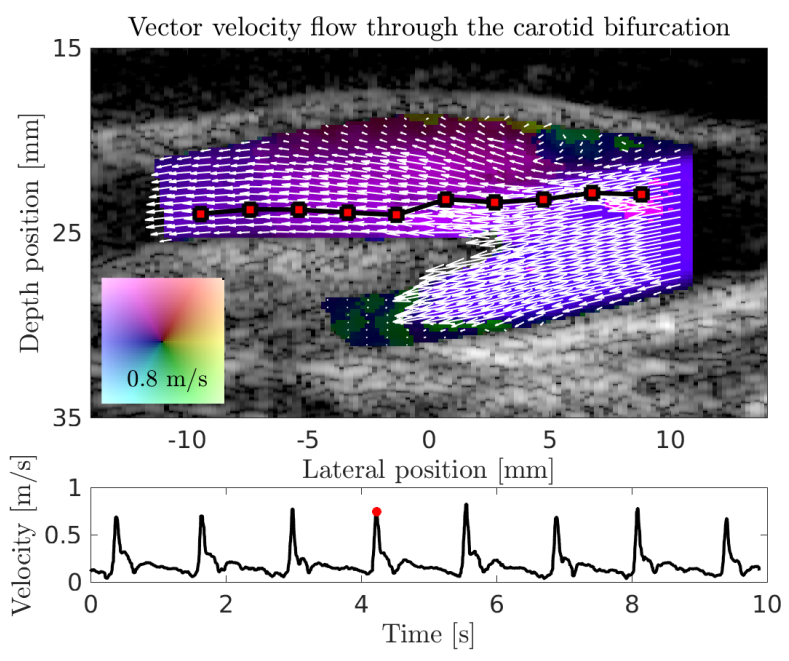

Fig. 9. In-vivo flow: Vector flow scan in a long axis view of the carotid bifurcation during peak systole. A streamline following the vector velocity field in the internal carotid artery at the level of the carotid bulb is also displayed.

\section{IN-VIVO RESULTS}

In-vivo measurements were carried out at the carotid bifurcation, and at the aortic valve of two healthy volunteers. The resulting vector flow images are shown in Figs. 9-10. The images were captured at peak systole, yielding values in the internal carotid artery at the level of the carotid bulb of roughly $0.8 \mathrm{~m} / \mathrm{s}$, and $1.5 \mathrm{~m} / \mathrm{s}$ of the left ventricular outflow tract across the aortic valve. The starting point of the superimposed streamlines were selected to start from the radial center of the vessels. Beneath the duplex images was the velocity waveform captured over the entire acquisition time. The consistent repetitive pattern over the different cardiac cycles is seen for the carotid scan. Only a quarter of a second was acquired from the cardiac scan - just enough to capture the peak systolic phase. The derived pressure changes found over the length of the streamlines are seen in Figs. 11 and 13. The temporal change in pressure differences between the starting- and end- 
Table I

EXPERIMENTAL RESULTS FROM THE $70 \%$ CONSTRICTED PHANTOM.

\begin{tabular}{l|ccc}
$70 \%$ constriction & Finite Element & Catheter & Ultrasound \\
$\pm 7 \mathrm{~mm}$ w. $15 \mathrm{~mL} / \mathrm{s}$ & & & \\
\hline Constant flow & $-5.4 \mathrm{kPa}$ & $-7.3 \mathrm{kPa} \pm 147 \mathrm{~Pa}$ & $-5.0 \mathrm{kPa} \pm 108 \mathrm{~Pa}$ \\
Pulsating flow & $-2.9 \mathrm{kPa}$ & $-5.4 \mathrm{kPa} \pm 52 \mathrm{~Pa}$ & $-3.0 \mathrm{kPa} \pm 57 \mathrm{~Pa}$ \\
\hline
\end{tabular}
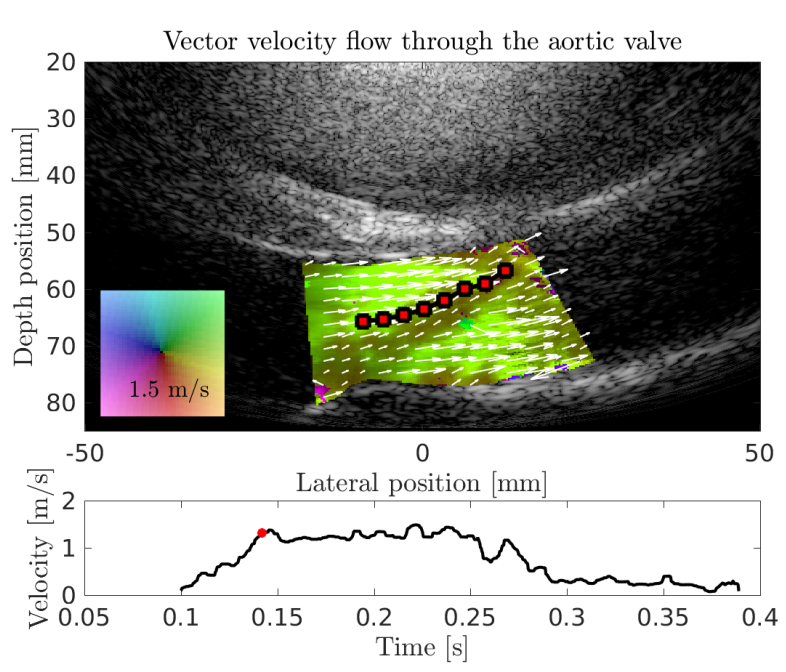

Fig. 10. In-vivo flow: Parastenal long axis view of vector flow of the left ventricular outflow tract across the aortic valve during peak systole.

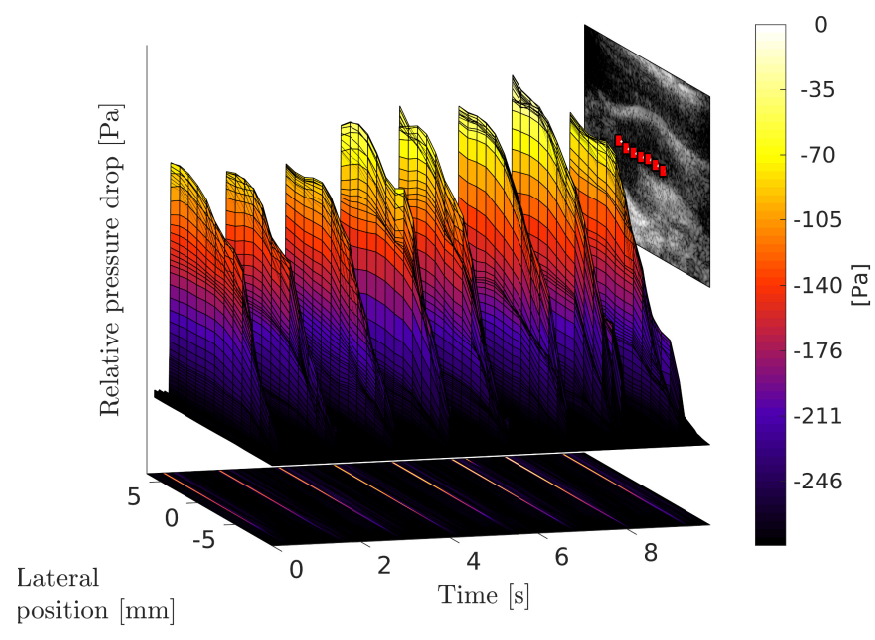

Fig. 11. In-vivo pressure: Changes in intravascular pressure measured as a function of time and longitudinal position along the streamline from the carotid scan. The estimated pressure drop is relative to the starting value at the beginning of the streamline, which is set to $0 \mathrm{~Pa}$.

point of the streamline in the carotid scan is shown in Fig. 12. The figure presents the mean pressure drop over the cycles, revealing a standard deviation of $6.6 \%$. Removing the flow cycle that contains the outlier seen $0.7 \mathrm{~s}$ into the flow cycle, reduces the standard deviation to $3.5 \%$ of the peak drop in pressure. The initial result from the aortic scan shows a peak pressure drop of roughly $-2 \mathrm{kPa}(-15.0 \mathrm{mmHg})$ across the valve.

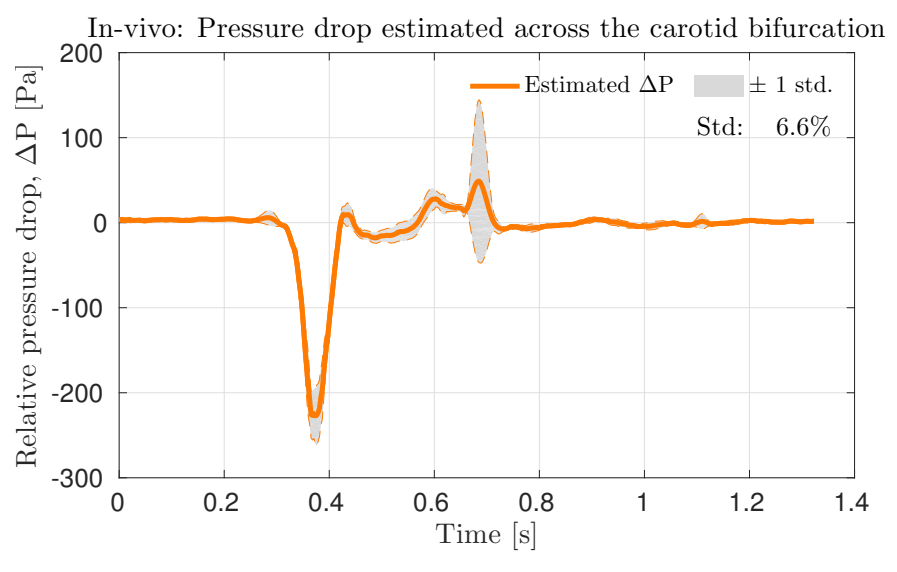

Fig. 12. Mean of the total pressure drop across the entire streamline in Fig. 9 for eight flow cycles. The peak pressure drop during peak systole is $-220 \mathrm{~Pa}$, with a standard deviation of $6.6 \%$.

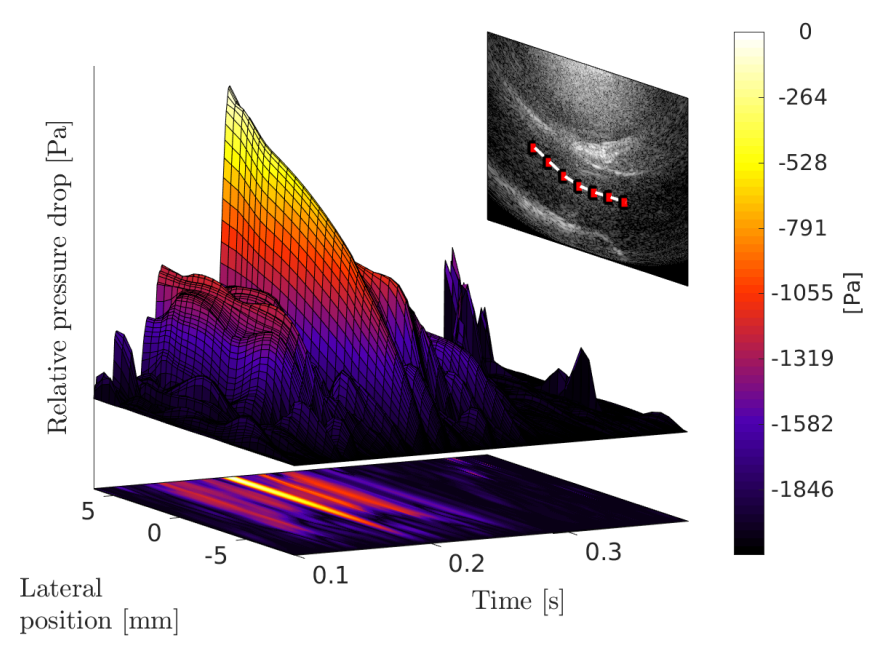

Fig. 13. In-vivo pressure: Changes in intravascular pressure measured as a function of time and longitudinal position along the streamline from the cardiac scan. The estimated pressure drop is relative to the starting value at the beginning of the streamline, which is set to $0 \mathrm{~Pa}$.

\section{DISCUSSION}

Non-invasive pressure changes have been calculated from vector velocity ultrasound data. The proposed approach was initially tested on simulated flow data through various models with different degrees of constrictions, before examined experimentally on a flow phantom. Pressure changes derived from the simulated velocity fields were compared with the reference pressure given by the FE model. The pressure changes derived from vector velocity ultrasound imaging were compared to results from both clinical pressure catheters and FE models. The study concluded by testing the feasibility of the method 
in-vivo over the carotid bifurcation and the aortic valve of two healthy volunteers.

Simulation: Applying the proposed estimator onto simulated flow fields showed convincing results with biases less than $2 \%$ for the $70 \%$ constricted flow model. Studying the bottom image of Fig. 4, further reveals that the bias arises downstream of the constriction, whereas excellent matches between the estimator's results and the reference model are seen in the upstream region. The reason for this is found in the factors that accounts for pressure loses in fluid flow: convective accelerations, viscous drag, and turbulent features. Only the first-mentioned is included in the presented study, which for the upstream part of a constriction is sufficient, as pressure changes here are mostly caused by forced convection of the flow. However, as the cross-sectional lumen of the geometry expands downstream, the flow starts to exhibit conditions where the effect of turbulent forces and viscous drag may become more prominent. This explains the discrepancy between the estimator's results and the FE model seen after the flow has passed the center of the constriction.

Phantom: Experimental pressure changes derived over the constricted phantom showed good agreements with the results obtained from CFD, having a bias of $7.1 \%$ for pulsatile flow. However, the importance of capturing the actual flow movements was clearly exemplified in case of the constant flow experiment, where the transducer was slightly off axis to the jet. Results from catheters consistently overestimated the pressure drop by at least $26 \%$ to the CFD model. This is equivalent to previous findings in the literature [2].

Results from the experimental study bring forward the greatest strengths, and the limitations of the proposed technique. Vector velocity imaging using high frame-rate approaches allows for tracking of streamlines with high precision due to large ensemble lengths. The high consistency of the employed flow estimator produced a basis for deriving pressure changes with standard deviations around $2 \%$, which is equivalent to the precision offered by the employed $4 \mathrm{~F}$ catheters. However, solely basing the proposed pressure estimator on measured velocity data makes it greatly sensitive to the magnitude of these, as the pressure scales directly with velocity squared.

Whether a frame-rate in the hundreds is needed for mapping the pressure gradients depends entirely of the flow conditions being investigated. In this study a frame-rate of $350 \mathrm{~Hz}$ was used, however, under less demanding flow conditions a lower frame-rate may be sufficient for capturing the pressure drop at an acceptable precision. Synthetic aperture imaging with directional beamforming allowed for detecting flow velocities as high as $3.0 \mathrm{~m} / \mathrm{s}$, while maintaining a wide field of view. Today, no commercially scanner is capable of displaying vector velocities of this magnitude without sacrificing the width of the interrogated flow region. This essentially hinders the possibility of detecting pressure gradients simultaneously across a region of interest.

In terms of millimeters of mercury, a pressure drop of $-3.0 \mathrm{kPa}$ to $-5.0 \mathrm{kPa}$ translates to roughly $-22 \mathrm{mmHg}$ to $37 \mathrm{mmHg}$, which for a clinically found stenotic vessel, is on the low side. In 1993, Donohue et al. [42] presented an in-vivo pressure catheter study showing that stenotic vessels with $50 \%-90 \%$ constrictions cause pressure drops from $-2 \mathrm{mmHg}$ to $-80 \mathrm{mmHg}$. Eight out of their 101 patients had stenoses of $70.1 \% \pm 14.7 \%$, for which drops in pressures of $-41.5 \pm 5.9 \mathrm{mmHg}$ were detected. Comparing these values with the results from the $70 \%$ constriction phantom reveal a discrepancy of a factor two. This discrepancy is likely a combination of catheter overestimation in their study, and the simplified vessel geometry examined in this study. Here, a flow model with a relatively short concentric stenosis was considered, where for the study by Donohue et al. clinically ill vessels were examined. The latter case rarely shows vessel curvatures as neat as the models designed in this study. Usually, highly irregular shapes are seen in actual arterial stenoses leading to more complex flow patterns, and thereby also a larger prevalence of turbulent flow features. Features such as eddies, localized turbulence, and jets that all contribute to the dissipation of energy will accelerate an increasing drop in pressure as the fluid passes through the stenotic region [43], [44]. The influences of these features are not included in the model presented in this study. Future work therefore includes measuring on more irregular geometries with increasing constriction-rates and lengths to investigate the application range of the suggested method, as flow approaches more turbulent conditions.

In-vivo: Results from the in-vivo scans demonstrated the feasibility of measuring pressure changes from 2-D vector velocities. The $10 \mathrm{~s}$ scan at the carotid bifurcation gave eight flow cycles, from where changes in pressure were estimated with a standard deviation of $6.6 \%$. This indicates an improvement in precision compared with the catheter study by Donohue et al. [42] that showed standard deviations of $14.2 \%$ for a $70 \%$ stenosis. The non-invasive nature of ultrasound allowed us to measure in the carotid artery, an area otherwise avoided by catheters, as direct contact with the arterial walls could make vulnerable plaques rupture causing cerebral infarctions [45].

For the cardiac scan, the acquisition rate was lowered to accommodate the longer travel time of the pulse. Further, only three low-resolution images were summed to cope with the high velocities across the heart valves. This led to difficulties in the velocity estimator as the signal-to-noise ratio dropped drastic. However, the imaging scheme could potentially be optimized using multi frequency encoding or coded excitations, thereby increasing the energy level while keeping the framerate constant. The result of the cardiac scan (Fig. 10) shows that the use of vector velocity flow enables new scan angles for examining the heart, as the operator becomes independent of the flow moving in the direction of the insonifying beam as shown in a previous study on vector velocity imaging [46] Measurements of the flow across the aortic valve was obtained in the parasternal long axis view as seen in Fig. 10, which in conventional echocardiography only is used for B-mode imaging due to the 90 degree insonation angle.

\section{Limitations}

The presented technique studies flow patterns of moving particles, from which the pressure, forcing the particles to move, is predicted. This makes the technique vulnerable to the flow estimator's capability to detect the true movement 
of the particles. The proposed model uses a 2-D velocity estimator approach, meaning that the out-of-plane motion gets excluded from the pressure algorithm. Whether or not this is acceptable, is greatly dependent on the complexity of the flow being examined. The influence of excommunicating the out-ofplane velocity component is minimized by positioning the probe parallel to the majority of flow movement. In clinical settings this is sought achieved by capturing the longitudinal direction of the vessel wall inside the 2-D scan plane. The correctness of this can be discussed, as blood in tortuous vessels seldom flows parallel to the vessel wall. Despite the crude assumption, this approach remains to be part of today's clinical practice when examining 3-D flow using a 1- or 2-D ultrasound flow system.

Deriving pressure changes from flow estimates is sensitive to biases in the flow estimator, as the pressure scales to the detected velocity squared. This also explains why the current way of determining pressure changes from a single Doppler estimate is extremely sensitive to the examiners ability to anglecorrect the flow. With 2-D flow estimation this becomes less sensitive to the operator's positioning of the probe, whereas 3D flow estimation would be ideal for mapping the intravascular pressure changes. It would therefore be highly interesting to see how this method performs with some of the recent developed 3-D ultrasound flow estimators [47]-[50].

The influence of viscosity has been neglected in this study, as flow in larger vessels was studied. This was considered valid, as the Reynolds number found in the flow case most sensible to viscous forces were calculated to 2000. However, moving into smaller vessels with lower Reynolds number, makes this assumption invalid.

Another disadvantage of the method is the reliance on detecting uninterrupted streamlines across the entire region of interest. In severely calcified vessels shadowing of the ultrasound image will occur. This conflicts with the proposed estimators ability to derived the trans-lesional pressure drop.

\section{Advantages}

Introduction of a 2-D technique that may replace the current 1-D ultrasound approach, or invasive catheters, has a wide avenue of advantages. Unlike the 1-D flow estimator, the presented method is independent of scan angle. This means that the technique can be employed along streamlines in areas where blood flow perpendicular to the ultrasound beam, as for instance in the carotid arteries. Further, the non-invasive nature of the proposed technique allows for the possibility of performing routine examinations for screening purposes, thus, expanding the application range of pressure sensing techniques. For instance, in echocardiographic pressure measurements across cardiac valves are often used in assessment of cardiac function, and valvular disease. With the proposed technique, a new approach for cardiac pressure assessment is introduced.

\section{CONCLUSION}

A non-invasive method for deriving intravascular pressure differences using 2-D vector velocity ultrasound data was presented. Initially, velocity data with a known pressure field were simulated for testing the performance of the estimator This showed a bias of less than $2.0 \%$. The simulation study was followed by experimental measurements conducted on a fabricated flow phantom with a concentric constriction of $70 \%$. Here, the estimated pressure changes during peak systole was $-3.0 \mathrm{kPa} \pm 57 \mathrm{~Pa}(-22.5 \mathrm{mmHg} \pm 0.4 \mathrm{mmHg})$ with a bias of $7.1 \%$ compared to FE simulations. Results from an in-vivo scan at the carotid bifurcation demonstrated the feasibility of measuring pressure changes along streamlines using 2-D ultrasound data. An acquisition of $10 \mathrm{~s}$ was made at the bifurcation, giving a pressure drop of $-227.2 \mathrm{~Pa} \pm 6.6 \%$. The result indicates that non-invasive 2-D velocity ultrasound estimation allows for exploring areas that lie beyond the reach of clinically employed catheters, and with better measurement accuracy.

\section{AcKnowledgments}

The presented work has been financially supported by grant 024-2008-3 from the Danish Advanced Technology Foundation, BK Medical Aps, Denmark and Radiometer A/S, Denmark.

\section{REFERENCES}

[1] D. S. Baim and W. Grossman, Grossman's cardiac catheterization angiography, and intervention. Lippincott Williams \& Wilkins, 2000.

[2] A. de Vecchi, R. E. Clough, N. R. Gaddum, M. C. M. Rutten, P. Lamata, T. Schaeffter, D. A. Nordsletten, and N. P. Smith, "Catheter-induced errors in pressure measurements in vessels: An in-vitro and numerical study," IEEE Trans. Biomed. Eng., vol. 61, no. 6, pp. 1844-1850, 2014

[3] J. J. Cavendish, L. I. Carter, and S. Tsimikas, "Recent advances in hemodynamics: Noncoronary applications of a pressure sensor angioplasty guidewire," Catheter Cardiovasc Interv, vol. 71, no. 6, pp. 748-758, 2008.

[4] W. M. Fairbank and M. O. Scully, "A new noninvasive technique for cardiac pressure measurements: resonant scattering of ultrasound from bubbles," IEEE Trans. Biomed. Eng., vol. 24, pp. 107-110, 1977.

[5] A. Bouakaz, P. J. Frinking, N. de Jong, and N. Bom, "Noninvasive measurement of the hydrostatic pressure in a fluid-filled cavity based on the disappearance time of micrometer-sized free gas bubbles," Ultrasound Med. Biol., vol. 25, pp. 1407-1415, 1999.

[6] W. T. Shi, F. Forsberg, J. S. Raichlen, and L. Needleman, "Pressure dependence of subharmonic signals from contrast microbubbles," Ultrasound Med. Biol., vol. 25, pp. 275-283, 1999.

[7] D. Adam, M. Sapunar, and E. Burla, "On the relationship between encapsulated ultrasound contrast agent and pressure," Ultrasound Med. Biol., vol. 31, pp. 673-686, 2005.

[8] F. Forsberg, J.-B. Liu, W. T. Shi, J. Furuse, M. Shimizu, and B. B Goldberg, "In vivo pressure estimation using subharmonic contrast microbubble signals: Proof of concept," IEEE Trans. Ultrason., Ferroelec., Freq. Contr., vol. 52, no. 4, pp. 581-583, 2005.

[9] J. Holen, R. Aaslid, and K. Landmark, "Determination of pressure gradient in mitral stenosis with a non-invasive ultrasound Doppler technique," Acta med. scand., vol. 32, pp. 455-460, 1976.

[10] E. M. Rohren, M. A. Kliewer, B. A. Carroll, and B. S. Hertzberg, "A spectrum of Doppler waveforms in the carotid and vertebral arteries," Am. J. Roentgenology, vol. 181, no. 6, pp. 1695-1704, 2003.

[11] A. L. Strauss, F. J. Roth, and H. Rieger, "Noninvasive assessment of pressure gradients across iliac artery stenoses: duplex and catheter correlative study," J. Ultrasound Med., vol. 12, no. 1, pp. 17-22, 1993.

[12] H. Baumgartner, H. Schima, G. Tulzer, and P. Kühn, "Effect of stenosis geometry on the Doppler-catheter gradient relation in vitro: A manifestation of pressure recovery," J. Am. Coll. Cardiol., vol. 21, no. 4, pp. 1018-1025, 1993.

[13] H. Baumgartner, T. Stefenelli, J. Niederberger, H. Schima, and G. Maurer, "Overestimation of catheter gradients by Doppler ultrasound in patients with aortic stenosis: A predictable manifestation of pressure recovery," $J$. Am. Coll. Cardiol., vol. 33, no. 6, pp. 1655-1661, 1999.

[14] M. J. Garcia, J. D. Thomas, and A. L. Klein, "New Doppler echocardiographic applications for the study of diastolic function," J. Am. Coll. Cardiol., vol. 32, no. 4, pp. 865-875, 1998. 
[15] N. L. Greenberg, P. M. Vandervoort, M. S. Firstenberg, M. J. Garcia, and J. D. Thomas, "Estimation of diastolic intraventricular pressure gradients by Doppler M-mode echocardiography," Am. J. Physiol. Heart Circ. Physiol., vol. 280, no. 6, pp. H2507-H2515, 2001.

[16] J. Bermejo, J. C. Antoranz, I. G. Burwash, J. L. Alvarez, M. Moreno, M. A. Garcia-Fernandez, and C. M. Otto, "In-vivo analysis of the instantaneous transvalvular pressure difference-flow relationship in aortic valve stenosis: implications of unsteady fluid-dynamics for the clinical assessment of disease severity," J. Heart Valve Dis., vol. 11, no. 4, pp. 557-566, 2002.

[17] D. Garcia, J. G. Dumesnil, L.-G. Durand, L. Kadem, and P. Pibarot, "Discrepancies between catheter and Doppler estimates of valve effective orifice area can be predicted from the pressure recovery phenomenon," J. Am. Coll. Cardiol., vol. 41, no. 3, pp. 435-442, 2003.

[18] A. K. Reddy, G. E. Taffet, S. Madala, L. H. Michael, M. L. Entman, and C. J. Hartley, "Noninvasive blood pressure measurement in mice using Doppler ultrasound," Ultrasound Med. Biol., vol. 29, no. 3, pp. 379-385, 2003.

[19] S. Ohtsuki and M. Tanaka, "Doppler pressure field deduced from the Doppler velocity field in an observation plane in a fluid," Ultrasound Med. Biol., vol. 29, no. 10, pp. 1431-1438, 2003.

[20] J. M. Meinders and A. P. G. Hoeks, "Simultaneous assessment of diameter and pressure waveforms in the carotid artery," Ultrasound Med. Biol., vol. 30, no. 2, pp. 147-154, 2004.

[21] R. Yotti, J. Bermejo, J. C. Antoranz, J. L. Rojo-Alvarez, C. Allue, J. Silva, M. M. Desco, M. Moreno, and M. A. Garcia-Fernandez, "Noninvasive assessment of ejection intraventricular pressure gradients," J. Am. Coll. Cardiol., vol. 43, no. 9, pp. 1654-1662, 2004.

[22] J. D. Thomas and Z. B. Popovic, "Intraventricular pressure differences a new window into cardiac function," Circulation, vol. 112, pp. 1684-1686, 2005.

[23] — "Assessment of left ventricular function by cardiac ultrasound," $J$ Am. Coll. Cardiol., vol. 48, no. 10, pp. 2012-2025, 2006.

[24] D. Garcia, P. Pibarot, and L.-G. Durand, "Analytical modeling of the instantaneous pressure gradient across the aortic valve," J. Biomech. vol. 38, pp. 1303-1311, 2005.

[25] B. W. A. M. M. Beulen, N. Bijnens, G. G. Koutsouridis, P. J. Brands, M. C. M. Rutten, and F. N. van de Vosse, "Toward noninvasive blood pressure assessment in arteries by using ultrasound," Ultrasound Med. Biol., vol. 37, no. 5, pp. 788-797, 2011.

[26] North American Symptomatic Carotid Endarterectomy Trial Collaborators, "Beneficial effect of carotid endarterectomy in symptomatic patients with high-grade carotid stenosis," N. Engl. J. Med, vol. 325, no. 7, pp. 445-453, 1991.

[27] L. Prandtl, Essentials of Fluid Dynamics. London: Blackie \& Son, 1952.

[28] N. B. Wood, "Aspects of fluid dynamics applied to the large arteries," $J$. Theor. Biol., vol. 199, no. 953, pp. 137-161, 1999.

[29] J. D. Thomas and A. E. Weyman, "Numerical modeling of ventricular filling," Ann. Biomed. Eng., vol. 20, pp. 19-39, 1992.

[30] H. Baumgartner, J. Hung, J. Bermejo, J. B. Chambers, A. Evangelista, B. P. Griffin, B. Iung, C. M. Otto, P. A. Pellikka, and M. Quinones, "Echocardiographic assessment of valve stenosis: EAE/ASE recommendations for clinical practice," European Journal of Echocardiography, pp. 1-25, 2009

[31] F. Donati, S. Myerson, M. M. Bissell, N. P. Smith, S. Neubauer, M. J. Monaghan, D. A. Nordsletten, and P. Lamata, "Beyond Bernoulli Improving the accuracy and precision of noninvasive estimation of peak pressure drops," Circ Cardiovasc Imaging, pp. 1-9, 2017.

[32] J. J. Charonko, C. V. King, B. L. Smith, and P. P. Vlachos, "Assessment of pressure field calculations from particle image velocimetry measurements," Meas. Sci. Technol., vol. 21, no. 10, p. 105401, 2010.

[33] S. M. Song, R. M. Leahy, D. P. Boyd, B. H. Brundage, and S. Napel, "Determining cardiac velocity fields and intraventricular pressure distribution from a sequence of ultrafast CT cardiac images," IEEE Trans. Med. Imag., vol. 13, no. 2, pp. 386-397, 1994.

[34] G.-Z. Yang, P. J. Kilner, N. B. Wood, S. R. Underwood, and D. N Firmin, "Computation of flow pressure fields from magnetic resonance velocity mapping," Magn. Reson. Med., vol. 36, no. 4, pp. 520-526, 1996.

[35] J. M. Tyszka, D. H. Laidlaw, J. W. Asa, and J. M. Silverman, "Threedimensional, time-resolved (4D) relative pressure mapping using magnetic resonance imaging," J. Magn. Reson. Imaging, vol. 12, no. 2, pp. 321-329, 2000.

[36] S. S. M. Lai, B. Y. S. Yiu, A. K. K. Poon, and A. C. H. Yu, "Design of anthropomorphic flow phantoms based on rapid prototyping of compliant vessel geometries," Ultrasound Med. Biol., vol. 39, no. 9, pp. 1654-1664, 2013.

[37] D. R. Enzmann, M. R. Ross, M. P. Marks, and N. J. Pelc, "Blood flow in major cerebral arteries measured by phase-contrast cine MR," Am. $J$. Neuroradiol., vol. 15, pp. 123-129, 1994.

[38] C. A. Villagomez-Hoyos, M. B. Stuart, K. L. Hansen, M. B. Nielsen, and J. A. Jensen, "Accurate angle estimator for high frame rate 2-D vector flow imaging," IEEE Trans. Ultrason., Ferroelec., Freq. Contr., vol. 63 , no. 6 , pp. 842-853, 2016.

[39] J. A. Jensen and S. I. Nikolov, "Directional synthetic aperture flow imaging," IEEE Trans. Ultrason., Ferroelec., Freq. Contr., vol. 51, pp. 1107-1118, 2004

[40] J. A. Jensen, H. Holten-Lund, R. T. Nilsson, M. Hansen, U. D. Larsen, R. P. Domsten, B. G. Tomov, M. B. Stuart, S. I. Nikolov, M. J. Pihl, Y. Du, J. H. Rasmussen, and M. F. Rasmussen, "SARUS: A synthetic aperture real-time ultrasound system," IEEE Trans. Ultrason., Ferroelec., Freq. Contr., vol. 60, no. 9, pp. 1838-1852, 2013

[41] C. A. Villagomez-Hoyos, M. B. Stuart, T. Bechsgaard, M. B. Nielsen, and J. A. Jensen, "High frame rate synthetic aperture vector flow imaging for transthoracic echocardiography," in Proc. SPIE Med. Imag., 2016.

[42] T. J. Donohue, M. J. Kern, F. V. Aguirre, R. G. Bach, T. Wolford, C. A Bell, and J. Segal, "Assessing the hemodynamic significance of coronary artery stenosis: Analysis of translesional pressure-flow velocity relations in patients," J. Am. Coll. Cardiol., vol. 22, no. 2, pp. 449-458, 1993.

[43] D. F. Young, N. R. Cholvin, and A. C. Roth, "Pressure drop across artificially induced stenoses in the femoral arteries of dogs," Circ Res., vol. 36, pp. 735-743, 1975 .

[44] K. L. Gould, "Quantification of coronary artery stenosis in vivo," Circ Res., vol. 57, no. 3, pp. 341-353, 1985.

[45] H. Al-Ameri, M. L. Thomas, A. Yoon, G. S. Mayeda, S. Burstein, R. A. Kloner, and D. M. Shavelle, "Complication rate of diagnostic carotid angiography performed by interventional cardiologists," Catheter Cardiovasc Interv., vol. 73, pp. 661-665, 2009.

[46] K. L. Hansen, H. Møller-Sørensen, J. Kjaergaard, M. B. Jensen, J. A Jensen, and M. B. Nielsen, "Aortic valve stenosis increases helical flow and flow complexity: A study of intra-operative cardiac vector flow imaging," Ultrasound Med. Biol., vol. 43, no. 8, pp. 1607-1617, 2017.

[47] M. J. Pihl, M. B. Stuart, B. G. Tomov, M. F. Rasmussen, and J. A. Jensen, "A transverse oscillation approach for estimation of three-dimensional velocity vectors. Part II: Experimental validation," IEEE Trans. Ultrason., Ferroelec., Freq. Contr, vol. 51, no. 10, pp. 1608-1618, 2014.

[48] M. Correia, J. Provost, M. Tanter, and M. Pernot, "4D ultrafast ultrasound flow imaging: in vivo quantification of arterial volumetric flow rate in a single heartbeat," Phys. Med. Biol., vol. 61, no. 23, pp. L48-L61, 2016.

[49] C. A. Villagomez-Hoyos, S. Holbek, M. B. Stuart, and J. A. Jensen, "High frame rate synthetic aperture 3D vector flow imaging," in Proc. IEEE Ultrason. Symp., 2016, pp. 1-4.

[50] S. Holbek, M. B. Stuart, and J. A. Jensen, "Volumetric 3-D vector flow measurements using a 62+62 row-column addressed array," in Proc. IEEE Ultrason. Symp., 2017, pp. 1-4. 